\title{
Some Understandings on Radial Motion at Transition in the Fermilab Booster
}

Xi Yang

March 20, 2007

\begin{abstract}
The transition crossing is space charge dominated in the Fermilab Booster. Since the longitudinal space charge forces are defocusing below transition and focusing above transition, they generate the mismatch at transition, which causes the longitudinal emittance growth above transition. It's proved by numerical simulation that such mismatch can be partially compensated by a particular radial motion at transition, which is operationally favored by the high intensity beam.
\end{abstract}

The transition crossing in Booster is space charge dominated. Usually, the nonlinear chromatic effect can cause the emittance growth during transition because particles with different energies cross transition at different times. The transition time is set by the synchronous particle; below transition, particles with positive energies relative to the synchronous particle become unstable since they are in the wrong phase, and above transition, particles with negative energies are unstable. The dependence of the transition energy upon the momentum deviation can be adjusted via different sextupole corrector settings such that the emittance growth due to the chromatic nonlinear effect can be greatly reduced. Fortunately, at the corrector setting of $\mathrm{I}_{\text {sextl }}=-97 \mathrm{~A}$ and $\mathrm{I}_{\text {sexts }}=97 \mathrm{~A}$, the dependence can be removed, see Figure 1. Space charge forces are mainly responsible for the longitudinal eimttance growth at transition. 


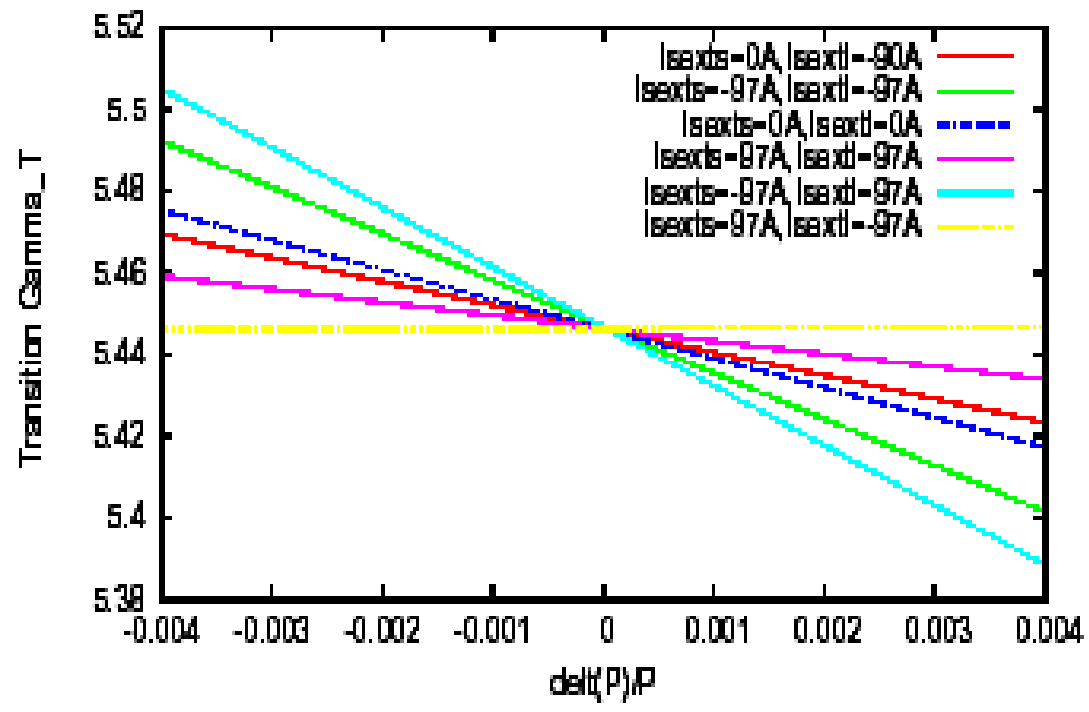

Figure1: Calculation of the dependence of transition energy upon momentum deviation at different sextupole corrector settings using MAD.

Since the longitudinal space charge force is always repulsive, it counteracts the rf focusing force below transition and enhances the focusing above transition. Right above transition, the bunch will appear with the larger bunch length left over from the influence of the space charge forces below transition; the equilibrium bunch length is now even shorter than that without space charge, the bunch will start to oscillate because of such mismatch at transition, see Figure 2. As the result, the non-linearities in the motion will cause particles with larger amplitudes to rotate more slowly leading to filamentation and dilution of the longitudinal emittance.

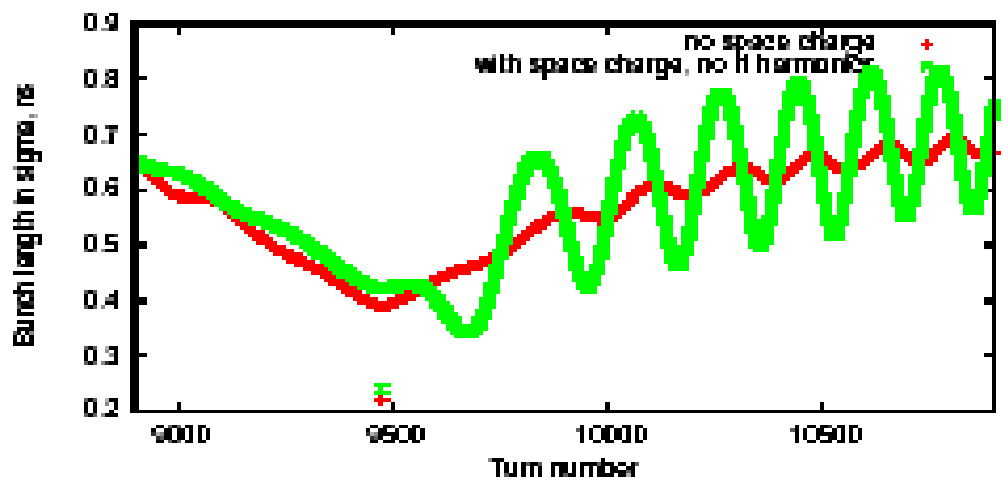

Figure 2: At the Booster batch intensity of 5e12, without (red) and with (green) space charge effect, bunch length in sigma vs. turn number is plotted. 
Voltage jump scheme from Valeri Lebedev uses the feed forward technique to compensate the space-charge induced mismatch at transition by increasing the rf focusing force right below transition [1]. Since the voltage jump scheme requires the rf system upgrade in Booster, it may take some time in implementing the hardware.

From the past experiences, Radial Motion is the key to reduce losses operationally at transition, especially for high intensity beams. Radial Motion requires the beam to be moved in radially right below transition and to be moved out radially right above transition.

Radial Motion can be achieved by decelerating or accelerating the beam relative to the synchronous particle. When the beam has less energy than the synchronous particle, it moves in radially, and vice visa. Right below transition, since the synchronous phase is in the range of $0^{\circ}$ to $90^{\circ}$, decelerating the beam relative to the synchronous particle is done by decreasing the synchronous phase, and at the same time, the beam gets more rf focusing to compensate the space charge defocusing forces. Right above transition, the synchronous phase is in the range of $90^{\circ}$ to $180^{\circ}$, accelerating the beam is done by decreasing the synchronous phase, and also, the beam gets less rf focusing to compensate the space charge focusing forces.

How much the space charge induced mismatch at transition can be compensated by Radial Motion depends upon the available aperture, see Figure 3, and the rest of the mismatch can be removed via quad damper above transition.

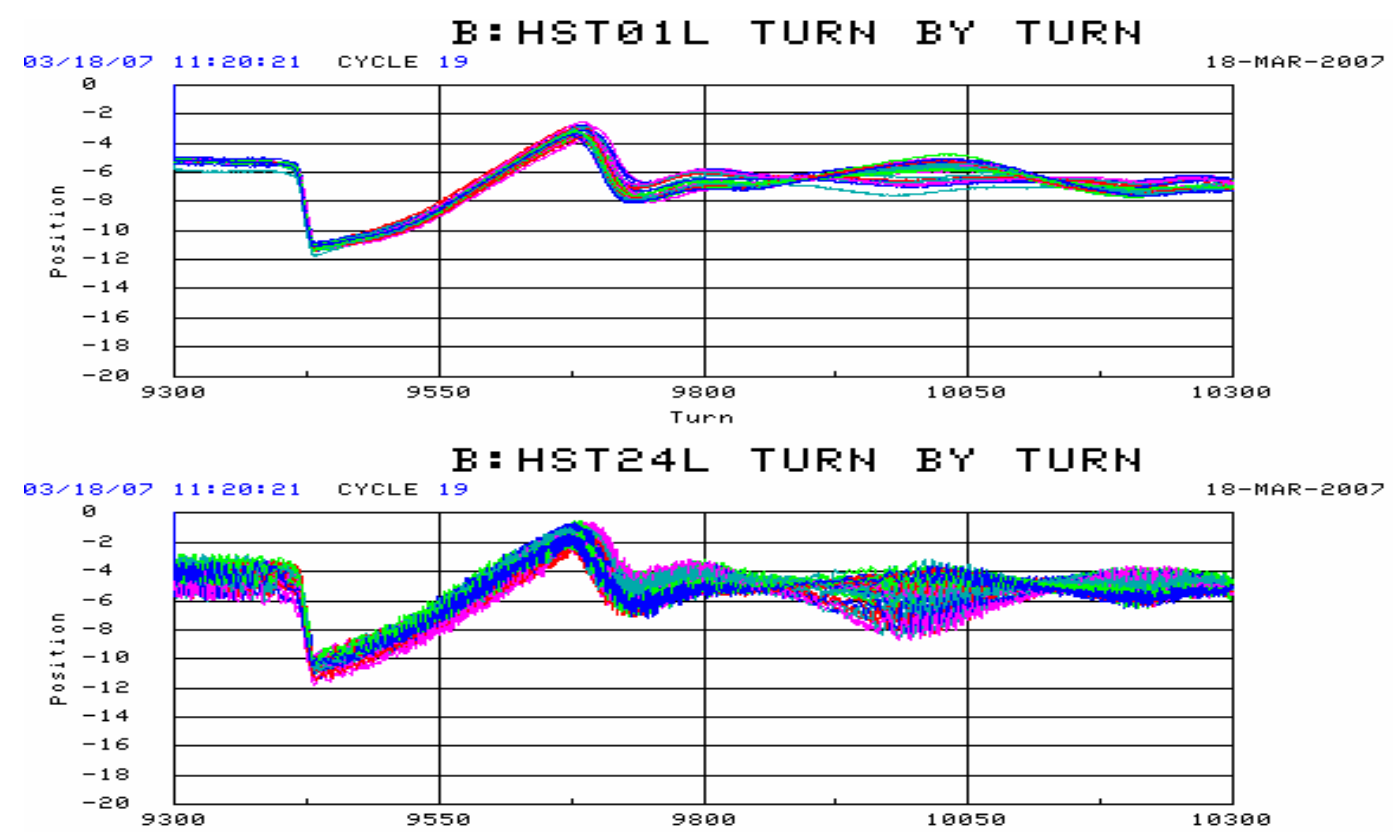


Figure 3: BPM turn by turn data taken at \$19 on March 18, 2007.

The available aperture is about 12 to14 $\mathrm{mm}$ at the batch size of 4.5e12, and it's used in the simulation. The ROF (radial offset) is optimized for the purpose of reducing the space charge induced mismatch; since the residual of such mismatch above transition is removed by the quad damper, more the mismatch is removed by Radial Motion (ROF bump), less the quad damper voltage is required, and better it is for the Booster rf stations. The results are shown in Figure 4. In the situation when the ROF bump is optimized at transition (green), less rf voltage is required by the quad damper.
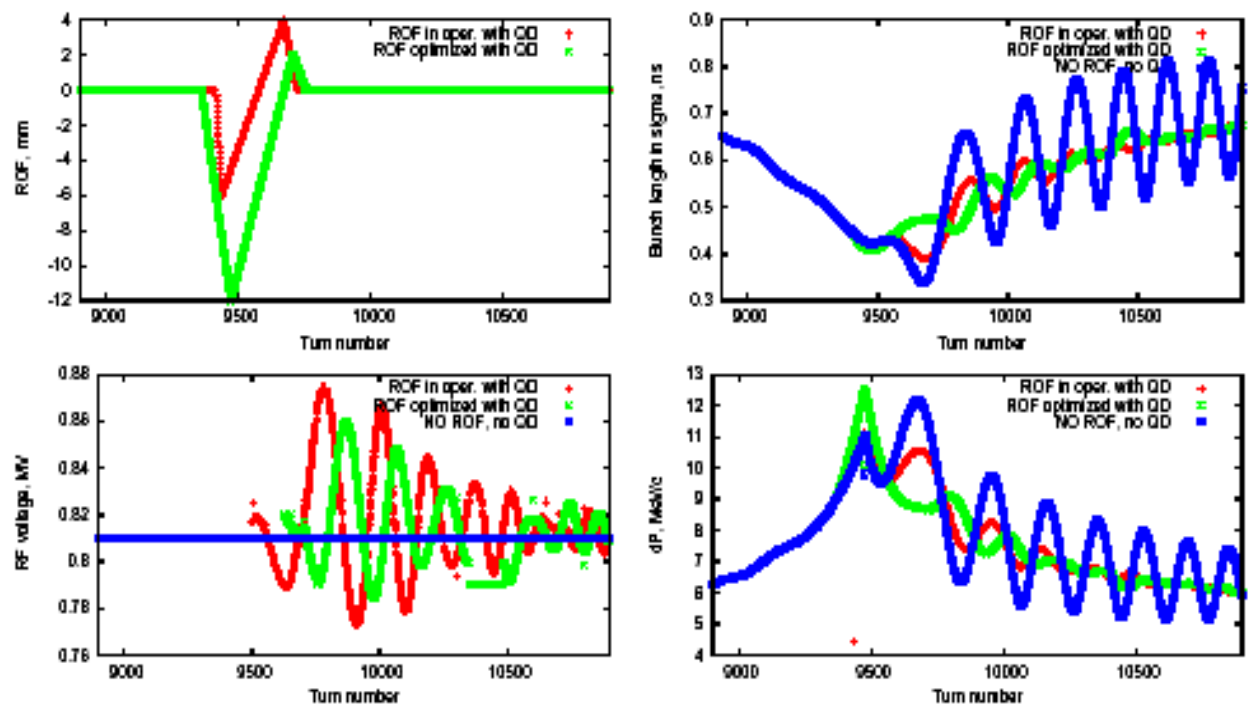

Figure 4 ROF, rf voltage (including the voltage feedback from quad damper), bunch length in sigma, and momentum spread vs. turn number are plotted from top to bottom and left to right for three cases, the red case with the operational ROF bump and the quad damper on, the green case with the optimal ROF bump and the quad damper on, and the blue case without any ROF bump and the quad damper off.

Using the optimized Radial Motion, which is the green case in Figure 4, the quad damper is turned on and off in order to find how much space charge induced mismatch is left above transition, as shown in Figure 5. Comparing quad damper on (red) and off (green), there is a small difference in the bunch length oscillation amplitudes above transition; once the ROF bump is removed (blue), the bunch length oscillates with a much larger amplitude above transition. 


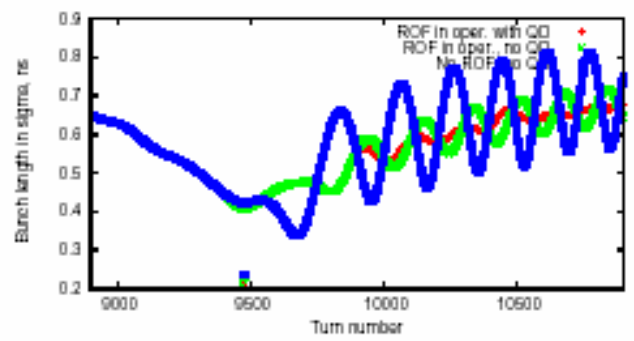

Figure 5 Bunch length vs. turn number is plotted for three cases, the red case with the optimal ROF bump and the quad damper on, the green case with the optimal ROF bump and the quad damper off, and the blue case without any ROF bump and the quad damper off.

The chromatic non-linear effect has much less influence on the transition compared to the space charge effect. A particular Radial Motion at transition provides the compensation to the space charge induced mismatch and makes a cleaner passage through transition. It reduces the bunch length oscillation after transition and at the same it reduces the quad damper voltage and makes the operation more reliable.

\section{References:}

- [1] V. A. Lebedev, “RF voltage jump”, Beams-doc-2690-v1. 